\title{
ON STEADY, LAMINAR TWO-DIMENSIONAL JETS IN COMPRESSIBLE VISCOUS GASES FAR BEHIND THE SLIT*
}

\author{
BY \\ M. Z. KRZYWOBLOCKI \\ University of Illinois
}

1. Introduction. Schlichting and Bickley $^{1}$ solved the problem of a laminar, steady, two-dimensional jet in an incompressible viscous fluid flowing through a narrow slit in a wall and then mixing with the surrounding medium at rest. In the present paper the problem of such a jet in a compressible viscous fluid is solved. The following equations are taken into account: equations of motion, continuity, energy and state. The coeffcients of viscosity and thermal conductivity are assumed to be functions of temperature. In order not to obscure the problem by many items of a simple algebraic nature this paper presents only an outline of the method of attack. The method enables one to find the distribution of the velocity, density etc. far behind the slit.

2. Basic equations. Assuming that the coefficients of viscosity $\mu$ and heat conductivity $K$ are variable functions, $\dagger$ one obtains the following equations (equation of motion, continuity, state and energy):

$$
\begin{gathered}
\rho\left[\mathrm{V}_{t}+\operatorname{grad}\left(\frac{1}{2} \mathrm{~V}^{2}\right)-(\mathrm{V} \times \omega)\right]=\rho \mathrm{F}-\operatorname{grad} p \\
-\frac{2}{3}(\operatorname{grad} \mu)(\operatorname{div} \mathrm{V})+2(\operatorname{grad} \mu \cdot \nabla) \mathrm{V}+(\operatorname{grad} \mu) \times \omega \\
+\frac{1}{3} \mu \operatorname{grad}(\operatorname{div} \mathrm{V})+\mu \nabla^{2} \mathrm{~V} \\
\rho_{t}+\nabla(\rho \mathrm{V})=0, \quad p=R \rho T, \\
J c_{,} \rho\left(T_{t}+\mathrm{V} \cdot \operatorname{grad} T\right)+p \operatorname{div} \mathrm{V} \\
=J[(\operatorname{grad} K) \cdot(\operatorname{grad} T)+K \operatorname{div}(\operatorname{grad} T)]+\phi, \\
\phi=\mu\left\{2 \nabla[(\mathrm{V} \cdot \nabla) \mathrm{V}]+\omega^{2}-2 \mathrm{~V} \cdot \operatorname{grad}(\operatorname{div} \mathrm{V})\right. \\
\left.-\frac{2}{3}(\operatorname{div} \mathrm{V})^{2}\right\}
\end{gathered}
$$

where $\omega=$ curl V.

${ }^{*}$ Received Aug. 10, 1948. This paper was presented to the American Mathematical Society on Feb. 28, 1948.

${ }^{1} \mathrm{H}$. Schlichting, Laminare Strahlausbreitung, Z. angew. Math. Mech., 13, 261-263 (1933). W. G. Bickley, The plane jet, The London, Edinburgh and Dublin Phil. Mag. (7) 23, 727-731 (1937). Also, Modern developments in fluid dynamics, ed. by S. Goldstein, Clarendon Press, Oxford, 1938. Bickley obtained the solution in the form of hyperbolic functions. Schlichting connected a series in ascending powers with an asymptotic solution, and obtained different numerical results.

$\dagger$ It is quite enough to assume that both $\mu$ and $K$ are functions of the temperature only and not of the pressure. Both functions may be assumed to be polynomials or power series in $T$ with properly selected coefficients (convergent series). 
Assume steady two-dimensional flow in rectangular coordinates without external forces, with the $z$-axis in the vertical direction and with the equation of state included in the equation of energy. The equations may be presented in the form:

$$
\begin{aligned}
& \rho u u_{x}-\mu u_{y y}=-p_{x}-\rho u_{y} v+\mu_{y} u_{y}+1 / 3 \mu v_{x y} \\
& +4 / 3\left(\mu u_{x}\right)_{x}-2 / 3 \mu_{x} v_{y}+\mu_{y} v_{x}, \\
& \rho u v_{x}-4 / 3 \mu v_{\nu \nu} \doteq-p_{\nu}-\rho v v_{\nu}+4 / 3 \mu_{\nu} v_{\nu}+1 / 3 \mu u_{x \nu} \\
& +\mu_{x} u_{y}-2 / 3 \mu_{y} u_{x}+\left(\mu v_{x}\right)_{x} \\
& u \rho_{x}=-\rho\left(u_{x}+v_{y}\right)-\rho_{y} v, \\
& J\left(c_{v} \rho u T_{x}-K T_{y y}\right)=-R \rho T\left(u_{x}+v_{y}\right)-J c_{r} \rho \nu T_{y} \\
& +J\left[\left(K T_{x}\right)_{x}+K_{y} T_{y}\right]+\phi, \\
& \phi=\mu\left\{4 / 3\left[u_{x}^{2}-u_{x} v_{y}+v_{y}^{2}\right]+\left(u_{y}+v_{x}\right)^{2}\right\},
\end{aligned}
$$

where subscripts denote partial differentiation.

A further assumption is that the jet cross sections taken into consideration are located very far downstream behind the slit. In an application of a series expansion in negative powers of $x$ this assumption enables one to neglect consistently the higher powers of $1 / x$. Consequently the expansion is an asymptotic one for large $x .^{*}$

A brief explanation of the method of solution of the set of differential equations will be given. The coefficients on the left-hand sides of those equations will be assumed so as to obtain ordinary linear differential equations of the second order. On the righthand sides the results from the preceding approximations will be used. The results will be represented in series:**

$$
u=\sum_{1}^{n} u_{i}, \quad v=\sum_{1}^{n} v_{i}, \quad \rho=\rho_{\infty}+\sum_{1}^{n} \rho_{i}, \quad T=T_{\infty}+\sum_{1}^{n} T_{i} .
$$

3. Initial approximation. Assume that the gas-jet flows through a narrow slit in the wall of height $h$. The velocity distribution across the cross section of the slit being constant, one may write: $M=h \rho_{0} u_{0}^{2}$, where $M$ denotes the rate at which the momentum flows across the initial section of the jet. This relation permits one to find the value $u_{0}$. The calculations given below will present the velocity, density, and temperature patterns across the jet at some distance from the slit. The value $u_{0}$ will serve as an auxiliary parameter.

\section{First approximation.}

(a) Longitudinal velocity component. Put $u_{1} \sim x^{-p} f(\eta), \eta \sim y x^{-a}$. Since the rate $M$ must be constant in all the cross sections, one has, in the first approximation,

*The applied method is, strictly speaking, an extension of Goldstein's method in incompressible flow. See: S. Goldstein, "On the Two-Dimensional Steady Flow of a Viscous Fluid Behind a Solid Body," Proc. Roy. Soc. of London, A, 142 (1933), p. 545-562.

**Throughout the paper the subscript zero denotes the values referring to the conditions inside the container from which the gas flows out. The subscript $\infty$ refers to the conditions in the undisturbed gas at rest outside the container. 


$$
M=2 \rho_{\infty} \int_{0}^{\infty} u_{1}^{2} d y \sim \int_{0}^{\infty} x^{-2 p} x^{a} f^{2}(\eta) d \eta
$$

Hence $2 p=q$. Another condition is that $u u_{1 x}$ must be of the same degree in $x$ as $u_{1 y y}$, or $p+1=p+2 q$. These two conditions give the values: $p=1 / 4, q=1 / 2$.

The longitudinal velocity component will always be calculated from the first equation of motion. In the first approximation put:* $v=0, \mu=\mu_{\infty}, \rho=\rho_{\infty}, u u_{x} \approx u_{0} u_{1 x}$ and neglect the variation of the pressure; i.e., $p_{x}=0$. In this approximation only the terms of degree $x^{-5 / 4}$ will be retained. One easily obtains

$$
\rho_{\infty} u_{0} u_{1 x}-\mu_{\infty} u_{1 y y}=0 .
$$

Introduce a non-dimensional coordinate $\eta$ :

$$
\begin{gathered}
\eta=y\left(\frac{u_{0}}{4 \nu_{\infty} x}\right)^{1 / 2}, \quad \nu_{\infty}=\frac{\mu_{\infty}}{\rho_{\infty}} ; \quad \eta_{x}=-\frac{1}{2} x^{-1} \eta, \quad \eta_{\nu}=\left(\frac{u_{0}}{4 \nu_{\infty} x}\right)^{1 / 2} ; \\
u_{1}=A_{1} u_{0} x^{-1 / 4} f_{1}(\eta) .
\end{gathered}
$$

The boundary conditions are that $u_{1}$ must tend to zero when $x \rightarrow \infty$, that $u_{1}$ must tend exponentially to zero when $y \rightarrow \infty$ and from the symmetry conditions that $u_{1}$ must be an even function of $y$, i.e., $u_{1 v}=u_{1 \eta}=0$ for $y=\eta=0$. After some elementary transformation one obtains

Put

$$
f_{1}^{\prime \prime}+2 \eta f_{1}^{\prime}+f_{1}=0 \text {. }
$$

and obtain

$$
f_{1}=k_{1} \exp \left(-\eta^{2}\right)
$$

$$
k_{1}^{\prime \prime}-2 \eta k_{1}^{\prime}-k_{1}=0 .
$$

This ordinary linear differential equation of the second order has a solution in the form of a series (see Appendix). The final form is

$$
u_{1}=A_{1} u_{0} x^{-1 / 4} k_{1} \exp \left(-\eta^{2}\right),
$$

with all the boundary conditions fulfilled.** The value of $A_{1}$ will be calculated from the condition that the rate $M$ must be constant in all the cross sections. Retaining from $k_{1}$ only one term gives:

$$
\begin{gathered}
M=4 A_{1}^{2} u_{0}^{3 / 2} \rho_{\infty} \nu_{\infty}^{1 / 2}\left(\frac{\pi}{2^{3}}\right)^{1 / 2} \quad \text { with } \quad \int_{0}^{\infty} \exp \left(-2 \eta^{2}\right) d \eta=\left(\frac{\pi}{2^{3}}\right)^{1 / 2} \\
A_{1}= \pm M^{1 / 2} u_{0}^{-1}\left(\frac{u_{0}}{2 \pi \mu_{\infty} \rho_{\infty}}\right)^{1 / 4} \text { in. } .^{1 / 4} * *
\end{gathered}
$$

*See the explanation below for the selection of $\rho_{\infty}$ instead of $\rho_{0}$.

**Another solution may be obtained by putting

$$
f_{1}=k_{12} \exp \left[-\eta^{2} / 2\right], \quad k_{12}^{\prime \prime}-\eta^{2} k_{12}=0 \text { (see appendix). }
$$

***Taking into account the second term will give the result:

$$
\left(\frac{1}{4}\right)\left[\int_{0}^{\eta} \exp \left(-\eta^{2}\right) d \eta-\eta \exp \left(-\eta^{2}\right)\right]
$$

This expression will add $25 \%$ to the value of $M$. 
(b) Transverse velocity component. This component will always be calculated from the second equation of motion. But in order to find the highest degree in $x$ of $v$, one calculates the value of $v_{t 1}$ from the continuity equation for the incompressible fluid: $u_{1 x}+v_{t 1 y}=0$, and obtains

$$
v_{t 1}=A_{2} u_{0} x^{-3 / 4} \int_{0}^{\eta}\left[\left(1-4 \eta^{2}\right) k_{1}+2 \eta k_{1}^{\prime}\right] \exp \left(-\eta^{2}\right) d \eta+C .
$$

The constant of integration $C$ must be equal to zero to fulfill the boundary conditions. The component $v$ must satisfy the following conditions: it must tend to zero when $x \rightarrow \infty$, it must tend exponentially to zero when $y \rightarrow \infty$, and from the symmetry conditions it must be an odd function of $y$ or $\eta$, i.e., $v=0$ for $y=\eta=0$. The final result is:

$$
\begin{aligned}
& v_{t 1}=A_{2} u_{0} x^{-3 / 4}\left[2 \eta k_{1} \exp \left(-\eta^{2}\right)-g_{t 1}\right] \\
& g_{t 1}=\int_{0}^{\eta} k_{1} \exp \left(-\eta^{2}\right) d \eta, \quad A_{2}=\frac{1}{2} A_{1}\left(\frac{\nu_{\infty}}{u_{0}}\right)^{1 / 2} \text { in. }^{3 / 4} .
\end{aligned}
$$

Although not all the boundary conditions are fulfilled, it is obvious that the value found for $v$ may be considered as the third approximation to $v\left(\sim x^{-3 / 4}\right)$. Hence $v_{1}=0$. $^{*}$

(c) Density. The density will always be calculated from the continuity equation. It must be equal to $\rho_{\infty}$ when $x \rightarrow \infty$ and $y \rightarrow \infty$. Hence let us put $\rho=\rho_{\infty}+\sum_{1}^{\infty} \rho_{i}$. On the left-hand side of the equation of continuity put the values $u \approx u_{0}, \rho=\rho_{\infty}+\rho_{1}$, and on the right-hand side the values $u_{1}, v_{1}$, and $\rho=\rho_{0}$. Retaining the terms of degree $x^{-5 / 4}$ one obtains: $u_{0} \rho_{1 x}=-\rho_{0} u_{1 x}$, or

$$
\rho_{1}=-\rho_{0} u_{0}^{-1} u_{1}+C=-A_{1} \rho_{0} x^{-1 / 4} k_{1} \exp \left(-\eta^{2}\right), \quad C=0 . * *
$$

The boundary conditions for $\rho_{1}$ are identical with those for $u_{1}$.

(d) Temperature. The temperature will always be calculated from the energy equation. In the first approximation only the terms of degree $x^{-5 / 4}$ will be retained. The temperature at infinity must be equal to $T_{\infty}$. Hence put $T=T_{\infty}+\sum_{1}^{\infty} T_{i}$. On the lefthand side of the energy equation put $\rho=\rho_{\infty}+\rho_{1}, u=u_{0}, T_{1} \sim x^{-1 / 4}, K=K_{\infty}$. On the right-hand side put the values $u_{1}, v_{1}, T=T_{0}, K=K_{\infty}, \rho=\rho_{\infty}+\rho_{1}$. The boundary conditions for $T_{1}$ are similar to those for $u_{1}$. One obtains

$$
J\left(c_{v} \rho_{\infty} u_{0} T_{1 x}-K_{\infty} T_{1 y y}\right)=-R \rho_{\infty} T_{0} u_{1 x} .
$$

Introduce a non-dimensional coordinate $\bar{\eta}$ :

$$
\begin{aligned}
& \bar{\eta}=y\left(\frac{c_{v} u_{0} \rho_{\infty}}{4 K_{\infty} x}\right)^{1 / 2}=B \eta, \quad B=\left(\frac{c_{v} \mu_{\infty}}{K_{\infty}}\right)^{1 / 2} ; \\
& \bar{\eta}_{x}=-\frac{1}{2} x^{-1} \bar{\eta}, \quad \bar{\eta}_{y}=\left(\frac{c_{v} u_{0} \rho_{\infty}}{4 K_{\infty} x}\right)^{1 / 2} ; \\
& \bar{T}_{1}=A_{3} T_{0} x^{-1 / 4} \bar{h}_{1}(\bar{\eta}), \quad A_{3}=\left(\frac{R}{J c_{v}}\right) A_{1}\left(\sim \text { in }^{1 / 4}\right) .
\end{aligned}
$$

${ }^{*}$ In order to verify whether the value $v_{1}=0$ fulfills the second equation of motion to the first degree of approximation, put into this equation on the left-hand side the values: $\rho=\rho_{\infty}, u=u_{0}, v=v_{1}$, $\mu=\mu_{\infty}$, and on the right-hand side the values: $p_{v}=0, v=0, u=u_{1}$. Retain only the terms of degree $x^{-5 / 4}$. One obtains: $\rho_{\infty} u_{0} v_{1 x}-(4 / 3) \mu_{\infty} v_{1 y y}=0$. Hence $v_{1}=0$ is really a solution.

${ }^{* *}$ It was assumed that $\rho_{1} \sim \rho_{0}$. 
After substituting (19) into (18) and performing the necessary transformations one obtains:

$$
\bar{h}_{1}^{\prime \prime}+2 \bar{\eta} \bar{h}_{1}^{\prime}+\bar{h}_{1}=4\left[\left(4 \eta^{2}-1\right) k_{1}-2 \eta k_{1}^{\prime}\right] \exp \left(-\eta^{2}\right) .
$$

Putting

$$
\bar{h}_{1}=\bar{p}_{1}(\bar{\eta}) \exp \left(-\bar{\eta}^{2}\right)
$$

one gets

$$
\left(\bar{p}_{1}^{\prime \prime}-2 \bar{\eta} \bar{p}_{1}^{\prime}-\bar{p}_{1}\right) \exp \left(-\bar{\eta}^{2}\right)=4\left[\left(4 \eta^{2}-1\right) k_{1}-2 \eta k_{1}^{\prime}\right] \exp \left(-\eta^{2}\right) .
$$

One complementary function of this equation is $\bar{k}_{1} ; *$ hence put $\bar{p}_{1}=\bar{k}_{1} \bar{r}_{1}$ and obtain

$$
\left[2\left(\bar{k}_{1}^{\prime}-\bar{\eta} \bar{k}_{1}\right) \bar{r}_{1}^{\prime}+\bar{k}_{1} \bar{r}_{1}^{\prime \prime}\right] \exp \left(-\bar{\eta}^{2}\right)=\bar{F}_{11},
$$

or

$$
\frac{d}{d \bar{\eta}}\left[\bar{k}_{1}^{2} \bar{r}_{1}^{\prime} \exp \left(-\bar{\eta}^{2}\right)\right]=\bar{F}_{11} \bar{k}_{1}
$$

Hence

$$
\bar{k}_{1}^{2} \bar{r}_{1}^{\prime} \exp \left(-\bar{\eta}^{2}\right)=\int_{0}^{\bar{\eta}} \bar{F}_{11} \bar{k}_{1} d \bar{\eta}+C=\bar{F}_{12} .
$$

As one may easily notice, after the integration is performed the expression on the righthand side will consist of the following functions: the error (probability) integral and $\sum_{0}^{\infty} \bar{\eta}^{(2 n+1)} \exp \left(-c \bar{\eta}^{2}\right)$. The constant of integration must be such as to fulfill the boundary condition $\bar{r}_{1}^{\prime}=0$ for $\bar{\eta}=0$. In the next step one obtains

$$
\bar{r}_{1}=\int_{0}^{\bar{\eta}} \bar{k}_{1}^{-2} \bar{F}_{12} \exp \left(\bar{\eta}^{2}\right) d \bar{\eta}+C_{1} .
$$

The constant of integration may be different from zero to fulfill the boundary condition for $\bar{\eta}=0$. $^{* *}$ The function $\bar{k}_{1}^{-2}$ may be expanded into a series by ordinary division. The function $\bar{r}_{1}$ will consist of the following functions: $\sum_{0}^{\infty} \bar{\eta}^{2 n} \exp \left(-c \bar{\eta}^{2}\right)$ and $\int_{0}^{\bar{\eta}} \bar{\eta}^{n}$ erf $(a \bar{\eta}) \exp \left(c \bar{\eta}^{2}\right) d \bar{\eta}$. This last integral is not tabulated and must be calculated in each particular case by numerical methods. One term in $\bar{r}_{1}$ will consist of an ordinary exponential function, which is not equal to zero for $\bar{\eta}=0$. $^{* * *}$ Hence all the boundary conditions are fulfilled and the final result is

$$
\begin{aligned}
& \overline{T_{1}}=A_{3} T_{0} x^{-1 / 4} \bar{k}_{1} \bar{r}_{1} \exp \left(-\bar{\eta}^{2}\right), \\
& T_{1}=A_{3} T_{0} x^{-1 / 4} k_{1} r_{1} \exp \left(-B^{2} \eta^{2}\right) .
\end{aligned}
$$

$C_{1}$ will be determined from the condition that with no heat dissipation from the jet the total heat content (enthalpy) expressed as mass $\times i=$ mass $\times c_{p}\left(T-T_{\infty}\right)=2 c_{\text {n }}$ $\int_{0}^{\infty} u_{1} T_{1}\left(\rho_{\infty}+\rho_{1}\right) d y=$ const., in each cross section.

*All the functions not included in the text are given in the Appendix.

${ }^{* *}$ In the present case it will be assumed that $C_{1} \neq 0$ (see below).

${ }^{* * *}$ Hence $\bar{r}_{1}(0) \neq 0$. But the value $T_{1}(0)$ will include $C_{1}$ (see below). 
(e). Coefficients of viscosity and heat conductivity. Assuming that $\mu$ can be expressed as $\mu=\bar{\mu}\left(1+a T+b T^{2}+\cdots\right)$ and $K$ as $K=K_{0} c_{p} \mu$, and substituting the value $T=$ $T_{\infty}+T_{1}$, one obtains

$$
\mu_{1}=\mu_{\infty}+\bar{\mu} \sum_{1}^{n} G_{2 i} T_{1}^{i}, \quad K_{1}=K_{0} c_{p} \mu_{1}
$$

\section{Second approximation.}

(a) Longitudinal velocity component. Put into the first equation of motion on the left-hand side the values $\rho=\rho_{\infty}+\rho_{1}, u \approx u_{0}+u_{2}, u_{x}=u_{1 x}+u_{2 x}, u_{y y}=u_{1 y y}+$ $u_{2 y y}, \mu=\mu_{1}$, and on the right-hand side the values $p_{x}=R(\rho T)_{x}, T=T_{\infty}+T_{1}$, $v=0$ and $u_{2} \sim x^{-1 / 2}$. In this approximation only the terms of degree $x^{-3 / 2}$ will be retained:

$$
u_{0} \rho_{\infty} u_{2 x}-\mu_{\infty} u_{2 y y}=\bar{\mu} G_{21}\left(T_{1} u_{1 y}\right)_{y}-u_{0} \rho_{1} u_{1 x}-R\left(\rho_{1} T_{1}\right)_{x} .
$$

All the functions have to be taken as functions of $\eta$. The solution of this equation will follow precisely the procedure outlined in the calculation of $T_{1}$. The main points are the following:

$$
\begin{gathered}
u_{2}=u_{0} x^{-1 / 2} f_{2}(\eta), \quad f_{2}=k_{2} \exp \left[-\frac{1}{2} \eta^{2}\right], \\
u_{0}^{2} \rho_{\infty} x^{-3 / 2}\left[f_{2}^{\prime \prime}+2\left(\eta f_{2}^{\prime}+f_{2}\right)\right]=u_{0}^{2} \rho_{\infty} x^{-3 / 2}\left[k_{2}^{\prime \prime}+\left(1-\eta^{2}\right) k_{2}\right] \exp \left[-\frac{1}{2} \eta^{2}\right]=F_{2} .
\end{gathered}
$$

One complementary function is $f_{21}=1+\sum_{i=2,4}^{\infty}, . b_{i} \eta^{i}$. Hence put $k_{2}=f_{21} g_{2}$ :

$$
\begin{gathered}
u_{0}^{2} \rho_{\infty} x^{-3 / 2}\left(2 f_{21}^{\prime} g_{2}^{\prime}+f_{21} g_{2}^{\prime \prime}\right) \exp \left[-\frac{1}{2} \eta^{2}\right]=F_{2}, \quad \frac{d}{d \eta}\left(f_{21}^{2} g_{2}^{\prime}\right)=\sum_{1}^{2} F_{2 i}, \\
g_{2}^{\prime}=f_{21}^{-2}\left[\int_{0}^{\eta} \sum F_{2 i} d \eta+A_{8}\right] .
\end{gathered}
$$

Because in the expression on the right-hand side of (31) there will appear terms of the form $\exp \left(-\eta^{2}\right)$, the constant $A_{8}$ must be chosen so as to make $g_{2}^{\prime}(0)=0$, where

$$
g_{2}=\int_{0}^{\eta}\left[f_{21}^{-2}\left(\int_{0}^{\eta} \sum F_{2 i} d \eta+A_{8}\right)\right] d \eta+A_{9} .
$$

Since the function $g_{2}$ will contain terms of the form exp $\left(-\eta^{2}\right)$, which do not vanish for $\eta=0$, the constant of integration $A_{9}$ must be calculated and is not equal to zero.* The result is

$$
u_{2}=u_{0} x^{-1 / 2} f_{21} g_{2} \exp \left[-\frac{1}{2} \eta^{2}\right],
$$

with all the boundary conditions fulfilled.

(b) Transverse velocity component. To verify the statement made above that $v_{2}=0$, put into the second equation of motion on the left-hand side the values $\rho=\rho_{\infty}+\rho_{1}$, $u \approx u_{0}+u_{2}, v=v_{2}, \mu=\mu_{1}$, and on the right-hand side the values $p_{v}=R(\rho T)_{\nu}$, $u=u_{1}+u_{2}, v=0, \mu=\mu_{1}$, and keep only the terms of degree $x^{-3 / 2}$ :

$$
3 u_{0} \rho_{\infty} v_{2 x}-4 \mu_{\infty} v_{2 y y}=0 .
$$

Hence $v_{2}=0$ is a solution.

${ }^{*}$ The rate $M$ with the previous value of $A_{1}$ and with $\rho=\rho_{\infty}+\rho_{1}, u=u_{1}+u_{2}$ must be constant in each cross section. This will give the value of $A_{9}$. 
(c) Density. Put into the equation of continuity on the left-hand side the values $u \approx u_{0}+u_{2}, \rho=\rho_{\infty}+\rho_{1}+\rho_{2}$, and on the right-hand side the values $\rho=\rho_{0}+\rho_{1}$, $u=u_{1}+u_{2}, v=0$. Preserving only the terms of degree $x^{-3 / 2}$, one obtains

$$
\begin{gathered}
u_{0} \rho_{2 x}=-\rho_{0} u_{2 x}-\rho_{1} u_{1 x}, \\
\rho_{2}=-\frac{1}{2} \rho_{0} x^{-1 / 2}\left\{2 f_{21} g_{2} \exp \left[-\frac{1}{2} \eta^{2}\right]-A_{1}^{2} k_{1}^{2} \exp \left(-2 \eta^{2}\right)\right\},
\end{gathered}
$$

with the constant of integration equal to zero and with all the boundary conditions fulfilled.

(d) Temperature. Put into the equation of energy on the left-hand side the values $\rho=\rho_{\infty}+\rho_{1}+\rho_{2}, u \approx u_{0}+u_{2}, T=T_{\infty}+T_{1}+T_{2}, K=K_{1}$, and on the righthand side the values $\rho=\rho_{\infty}+\rho_{1}+\rho_{2}, T=T_{\infty}+T_{1} ; u=u_{1}+u_{2}, v=0, \mu=\mu_{1}$. Retaining only the terms of degree $x^{-3 / 2}$ gives:

$$
\begin{aligned}
& J\left(c_{\nu} u_{0} \rho_{\infty} \bar{T}_{2 x}-K_{\infty} \bar{T}_{2 y y}\right)=J \bar{K} G_{21}\left(\bar{T}_{1} \bar{T}_{1 y}\right)_{\nu}+\mu_{\infty} \bar{u}_{1 y}^{2} \\
& \quad-J c_{\nu} u_{0} \bar{\rho}_{1} \bar{T}_{1 x}-R\left[\rho_{\infty}\left(\bar{u}_{1 x} \bar{T}_{1}+\bar{u}_{2 x} T_{\infty}\right)+\bar{u}_{1 x} \bar{\rho}_{1} T_{\infty}\right],
\end{aligned}
$$

where bars over the letters denote the functions of $\bar{\eta}$. Again the procedure is identical with that explained above:

$$
\begin{gathered}
\bar{T}_{2}=T_{0} x^{-1 / 2} \bar{h}_{2}(\bar{\eta}), \quad \bar{h}_{2}=\bar{p}_{2} \exp \left[-\frac{1}{2} \bar{\eta}^{2}\right], \\
A_{10} x^{-3 / 2}\left[\bar{h}_{2}^{\prime \prime}+2\left(\bar{\eta} \bar{h}_{2}^{\prime}+\bar{h}_{2}\right)\right]=A_{10} x^{-3 / 2}\left[\bar{p}_{2}^{\prime \prime}+\left(1-\bar{\eta}^{2}\right) \bar{p}_{2}\right] \exp \left[-\frac{1}{2} \bar{\eta}^{2}\right]=\bar{G}_{2} .
\end{gathered}
$$

With a complementary function $\bar{f}_{21}$ * the procedure identical to that in Sec. 5(a) gives

$$
\bar{f}_{21}^{2} \bar{r}_{2}^{\prime}=\sum_{i=1}^{6} B_{i} \bar{I}_{2 i}+B_{7} .
$$

The constant of integration $B_{7}$ must be so chosen as to make $\bar{r}_{2}^{\prime}(0)=0$, where

$$
\bar{r}_{2}=\int_{0}^{\bar{\eta}}\left(\sum_{i=1}^{6} B_{i} \bar{I}_{2 i}+B_{7}\right) \bar{f}_{21}^{-2} d \bar{\eta}+B_{8} .
$$

The constant $B_{8}$ is not equal to zero, hence $\bar{r}_{2}(0) \neq 0 .^{* *}$ Hence:

$$
\begin{aligned}
& \bar{T}_{2}=T_{0} x^{-1 / 2} \bar{f}_{21} \bar{r}_{2} \exp \left[-\frac{1}{2} \bar{\eta}^{2}\right], \\
& T_{2}=T_{0} x^{-1 / 2} f_{21} r_{2} \exp \left[-\frac{1}{2} B^{2} \eta^{2}\right],
\end{aligned}
$$

with all the boundary conditions fulfilled.

(e) Coefficients of viscosity and heat conductivity.

$$
\begin{gathered}
\mu_{2}=\mu_{\infty}+\bar{\mu}\left[G_{21}\left(T_{1}+T_{2}\right)+G_{31}\left(T_{1}^{2}+T_{2}^{2}\right)+G_{32} T_{1} T_{2}+\cdots\right], \\
K_{2}=K_{0} c_{p} \mu_{2} .
\end{gathered}
$$

*The function $\bar{f}_{21}$ is the function $f_{21}$ with $\eta$ changed to $\bar{\eta}$.

${ }^{* *} B_{8}$ will be determined from the condition that

$$
\operatorname{mass} i=2 c_{p} \int_{0}^{\infty}\left(\rho_{\infty}+\rho_{1}+\rho_{2}\right)\left(u_{1}+u_{2}\right)\left(T_{1}+T_{2}\right) d y=\text { const., }
$$

in each cross section. 


\section{Third approximation.}

(a) Longitudinal velocity component. Putting on the left-hand side of Eq. (4) the values $\rho=\rho_{\infty}+\rho_{1}+\rho_{2}, u \approx u_{0}+u_{2}+u_{3}, u_{x}=u_{1 x}+u_{2 x}+u_{3 x}, \mu=\mu_{2}, u_{y y}=$ $u_{1 y y}+u_{2 y y}+u_{3 y y}$, and on the right-hand side the values $p_{x}=R(\rho T)_{x}, v=0$, etc., and letting $u_{3} \sim x^{-3 / 4}$ gives the result:

$$
\rho_{\infty} u_{0} u_{3 x}-\mu_{\infty} u_{3 y y}=F_{3} .
$$

The procedure remains unchanged:

$$
\begin{gathered}
u_{3}=u_{0} x^{-3 / 4} f_{3}, \\
u_{0}^{2} \rho_{\infty} x^{-7 / 4}\left(f_{3}^{\prime \prime}+2 \eta f_{3}^{\prime}+3 f_{3}\right)=K_{3}, \\
f_{3}=k_{3} \exp \left[-\frac{1}{2} \eta^{2}\right], \quad u_{0}^{2} \rho_{\infty} x^{-7 / 4}\left[k_{3}^{\prime \prime}+\left(2-\eta^{2}\right) k_{3}\right] \exp \left[-\frac{1}{2} \eta^{2}\right]=K_{3} .
\end{gathered}
$$

One complementary function is $f_{31}$. Put $k_{3}=f_{31} g_{3}$ :

$$
\begin{gathered}
u_{0}^{2} \rho_{\infty} x^{-7 / 4} \frac{d}{d \eta}\left(f_{31}^{2} g_{3}^{\prime}\right) \exp \left[-\frac{1}{2} \eta^{2}\right]=f_{31} K_{3}, \\
f_{31}^{2} g_{3}^{\prime}=\sum_{1}^{6} I_{3 i}+C_{6}, \quad g_{3}=\int_{0}^{\eta}\left(\sum_{1}^{6} I_{3 i}+C_{6}\right) f_{31}^{-2} d \eta+C_{7} .
\end{gathered}
$$

$C_{6}$ must be chosen so as to give $g_{3}^{\prime}(0)=0 . C_{7}$ must be calculated and is not equal to zero. The result is:

$$
u_{3}=u_{0} x^{-3 / 4} f_{31} g_{3} \exp \left[-\frac{1}{2} \eta^{2}\right] .
$$

(b) Transverse velocity component. Put on the left-hand side of Eq. (5) the values $\rho=\rho_{\infty}+\rho_{1}+\rho_{2}, u \approx u_{0}+u_{1}+u_{2}, v=v_{3} \sim x^{-3 / 4}, \mu=\mu_{2}$, and on the right-hand side the values $p_{y}=R(\rho T)_{y}, u=u_{1}+u_{2}+u_{3}, v=0$. Retaining only the terms of degree $x^{-7 / 4}$ gives the result:

$$
3 \rho_{\infty} u_{0} v_{3 x}-4 \mu_{\infty} v_{3 y y}=\mu_{\infty} u_{1 x y} .
$$

One may easily show that $v_{3}=v_{t 1}$, given by (16), is a solution of (49). In this case $u_{1 x}=-v_{3 y}{ }^{*}$ or $u_{1 x y}=-v_{3 y y}$, hence Eq. (49) changes to $\rho_{\infty} u_{0} v_{3 x}-\mu_{\infty} v_{3 y y}=0$. But $v_{3}=-\int u_{1 x} d y,{ }^{* *}$ or $v_{3 x}=-\int u_{1 x x} d y$ and $v_{3 y y}=-\int u_{1 x y y} d y$. Hence after substituting into the simplified equation (49), i.e. $\rho_{\infty} u_{0} \int u_{1 x x} d y-\mu_{\infty} \int u_{1 x y y} d y$, differentiating with respect to $y$ and integrating with respect to $x$, one obtains exactly Eq. (8). But $v_{3}=$ $v_{t 1}$ does not fulfill the boundary conditions when $y \rightarrow \infty$, viz., $g_{t 1} \neq 0$ for $y \sim \eta \rightarrow \infty$. One has to find a solution of the homogeneous equation (49). To this end put

$$
v_{3}=u_{0} x^{-3 / 4} p_{3},
$$

and obtain $p_{3}^{\prime \prime}+(3 / 2) \eta p_{3}^{\prime}+(9 / 4) p_{3}=0$. One distinct solution of $(50)$ is the series $p_{31}$; another ${ }^{2}$ is

$$
p_{32}=p_{31} \int_{0}^{\eta} p_{31}^{-2} \exp \left[-\frac{3}{4} \eta^{2}\right] d \eta+C_{8} .
$$

*See Sec. 4(b).

**The constant of integration equals 0 .

2See: E. L. Ince, Ordinary differential equations, Longmans, Green and Co., New York, 1927, p. 122. 
An integration term by term may be applied. $C_{8}$ must be chosen so as to make $\left(g_{t 1}+p_{32}\right)=0$ as $\eta \rightarrow \infty$.

7. Final remarks. The approximations of higher degree are only a matter of routine work. In the theory of incompressible fluids equations of motion and continuity are used. The velocity components may be derived from the stream function. But it is almost impossible always to satisfy all the boundary conditions for all the velocity components. Usually the $v-$ component satisfies only one condition (for $\eta=0$ but not for $\eta \rightarrow \infty)$. It was shown above that in the theory of compressible flow one may always satisfy all the boundary conditions by use of the distinct solutions of homogeneous equations. This last remark refers equally to all the dependent variables.*

APPENDIX

4(a). $\quad k_{1}=1+a_{2} \eta^{2}+a_{4} \eta^{4}+\cdots+a_{2 n} \eta^{2 n}+\cdots, \quad a_{2}=1 / 2, \quad a_{4}=5 / 24$,

$a_{6}=1 / 16, \quad a_{8}=13 / 896, \quad a_{10}=221 / 80640, \quad$ etc.;

$k_{12}=1+\alpha_{4} \eta^{4}+\alpha_{8} \eta^{8}+\cdots, \quad \alpha_{4}=1 / 12, \quad \alpha_{8}=1 / 672, \quad$ etc.

$4(\mathrm{~d}) . \bar{k}_{1}=1+\sum_{i=2,4, \ldots}^{\infty} a_{i} \bar{\eta}^{i}, \quad \bar{F}_{11}=4\left[\left(4 B^{-2} \bar{\eta}^{2}-1\right) \bar{k}_{1}-2 \bar{\eta} \bar{k}_{1}^{\prime}\right] \exp \left(-B^{-2} \bar{\eta}^{2}\right)$.

4(e). $\quad \mu_{\infty}=\bar{\mu}\left(1+a T_{\infty}+b T_{\infty}^{2}+c T_{\infty}^{3}+\cdots\right), \quad G_{21}=a+2 b T_{\infty}+3 c T_{\infty}^{2}+\cdots$,

$G_{22}=b+3 c T_{\infty}+\cdots, \quad$ etc.; $\quad K_{\infty}=K_{0} c_{p} \mu_{\infty}$.

5(a). $\quad F_{2}=4\left[R\left(\rho_{1} T_{1}\right)_{x}+u_{0} \rho_{1} u_{1 x}-\bar{\mu} G_{21}\left(u_{1 y} T_{1}\right)_{v}\right], \quad b_{2}=-1 / 2, \quad b_{4}=1 / 8$,

$b_{6}=-1 / 48, \quad b_{8}=1 / 384, \quad b_{10}=-7 / 34560, \quad$ etc.;

$F_{21}=\sum_{1}^{4} F_{21 i} f_{21} \exp \left[-\left(B^{2}+1 / 2\right) \eta^{2}\right], \quad F_{211}=\left\{2\left(A_{5}+A_{7}\right)-4\left[A_{5}(1+B)\right.\right.$

$\left.\left.+A_{7}\right] \eta\right\} k_{1}^{2} r_{1}, \quad F_{212}=4\left(A_{5}+A_{7}\right) k_{1} k_{1}^{\prime} r_{1} \eta, \quad F_{213}=\left(2 A_{5} k_{1} r_{1}^{\prime} \eta-A_{7} k_{1}^{\prime \prime} r_{1}\right) k_{1}$,

$F_{214}=-A_{7}\left[\left(k_{1} r_{1}\right)_{\eta}-2 B^{2} \eta k_{1} r_{1}\right]\left(k_{1}^{\prime}-2 \eta k_{1}\right), \quad F_{22}=A_{6}\left[2 \eta k_{1}^{\prime}+\left(1-4 \eta^{2}\right) k_{1}\right] k_{1} f_{21}$

$\cdot \exp \left[(-3 / 2) \eta^{2}\right], \quad A_{5}=A_{1} A_{3} R u_{0}^{-2} T_{0}\left(\rho_{0} / \rho_{\infty}\right)\left(\mathrm{inch}^{1 / 2}\right)$,

$A_{6}=A_{1}^{2}\left(\rho_{0} / \rho_{\infty}\right)\left(\right.$ inch $\left.^{1 / 2}\right), \quad A_{7}=A_{1} A_{3} G_{21} T_{0} \bar{\mu} \mu_{\infty}^{-1}\left(\right.$ inch $\left.{ }^{1 / 2}\right)$.

5(d). $\bar{K}=K_{0} c_{p} \bar{\mu}, \quad A_{10}=J c_{v} u_{0} \rho_{\infty} T_{0}, \quad \bar{G}_{2}=4\left\{R\left[\rho_{\infty}\left(\bar{u}_{1 x} \bar{T}_{1}+\bar{u}_{2 x} T_{\infty}\right)+\bar{u}_{1 x} \bar{\rho}_{1} T_{\infty}\right]\right.$

$\left.+J\left[c_{v} u_{0} \bar{\rho}_{1} \bar{T}_{1 x}-\bar{K} G_{21}\left(\bar{T}_{1} \bar{T}_{1 y}\right)_{y}\right]-\mu_{\infty} \bar{u}_{1 y}^{2}\right\} ; \quad B_{1}=A_{1} A_{3} R\left(J c_{v}\right)^{-1}\left(\operatorname{inch}^{1 / 2}\right)$,

$B_{2}=R T_{\infty}\left(J c_{v} T_{0}\right)^{-1}($ dimensionless $), \quad B_{3}=A_{1}^{2} R \rho_{0} T_{\infty}\left(J c_{v} \rho_{\infty} T_{0}\right)^{-1}\left(\right.$ inch $\left.^{1 / 2}\right)$,

*This fact has been shown by the author in several papers. 


$$
\begin{aligned}
& B_{4}=A_{1} A_{3} \rho_{0} \rho_{\infty}^{-1}\left(\text { inch }^{1 / 2}\right), \quad B_{5}=A_{3}^{2} G_{21} \bar{K} T_{0} \rho_{0}\left(K_{\infty} \rho_{\infty}\right)^{-1}\left(\text { inch }^{1 / 2}\right) ; \\
& \bar{I}_{21}=\int_{0}^{\tilde{\eta}}\left[\left(4 B^{-2} \bar{\eta}^{2}-1\right) \bar{k}_{1}-2 \bar{k}_{1}^{\prime} \bar{\eta}\right] \bar{k}_{1} \bar{r}_{1} \bar{f}_{21} \exp \left[-\left(B^{-2}+1 / 2\right) \bar{\eta}^{2}\right] d \bar{\eta}, \\
& \bar{I}_{22}=2 \int_{0}^{\bar{\eta}}\left[\left(B^{-2} \bar{\eta}^{2}-1\right) \bar{f}_{21} \bar{g}_{2}-\bar{\eta}\left(\bar{f}_{21} \bar{g}_{2}\right)_{\tilde{\eta}}\right] \bar{f}_{21} \exp \left[(-1 / 2)\left(B^{-2}-1\right) \bar{\eta}^{2}\right] d \bar{\eta}, \\
& \bar{I}_{23}=\int_{0}^{\bar{\eta}}\left[2 \bar{k}_{1}^{\prime} \bar{\eta}+\left(1-4 B^{-2} \bar{\eta}^{2}\right) \bar{k}_{1}\right] \bar{f}_{21} \bar{k}_{1} \exp \left[-\left(2 B^{-2}-1 / 2\right) \bar{\eta}^{2}\right] d \bar{\eta}, \\
& \bar{I}_{24}=\int_{0}^{\bar{\eta}}\left[\bar{\eta}\left(\bar{k}_{1} \bar{r}_{1}\right)_{\tilde{\eta}}-\left(4 \bar{\eta}^{2}-1\right) \bar{k}_{1} \bar{r}_{1}\right] \bar{f}_{21} \bar{k}_{1} \exp \left[-\left(B^{-2}+1 / 2\right) \bar{\eta}^{2}\right] d \bar{\eta} \\
& \bar{I}_{25}=-\int_{0}^{\bar{\eta}}\left\{\left(\bar{k}_{1} \bar{r}_{1}\right)_{\bar{\eta} \bar{\eta}}-4 \bar{\eta}\left(\bar{k}_{1} \bar{r}_{1}\right)_{\dot{\eta}}+\left(4 \bar{\eta}^{2}-1\right) \bar{k}_{1} \bar{r}_{1}+\left[\left(\bar{k}_{1} \bar{r}_{1}\right)_{\bar{\eta}}-2 \bar{\eta} \bar{k}_{1} \bar{r}_{1}\right]^{2}\right\} \bar{f}_{21} \\
& \cdot \exp \left[-3 \bar{\eta}^{2} / 2\right] d \bar{\eta} \\
& \bar{I}_{26}=-\int_{0}^{\bar{\eta}}\left(\bar{k}_{1}^{\prime}-2 B^{-2} \bar{\eta} \bar{k}_{1}\right)^{2} \bar{f}_{21} \exp \left[-\left(2 B^{-2}-1 / 2\right) \bar{\eta}^{2}\right] d \bar{\eta} \\
& G_{31}=b+3 c T_{\infty}+\cdots, \quad G_{32}=2 b+6 c T_{\infty}+\cdots
\end{aligned}
$$

6(a). $\quad F_{3}=\bar{\mu}\left[G_{21}\left(u_{1 y} T_{2}+u_{2 y} T_{1}\right)+G_{31} u_{1 y} T_{1}^{2}\right]_{y}-u_{0}\left(u_{1 x} \rho_{2}+u_{2 x} \rho_{1}\right)-\rho_{\infty} u_{1 x} u_{2}$

$$
-R\left(\rho_{1} T_{2}+\rho_{2} T_{1}\right)_{x}, \quad K_{3}=-4 F_{3} ; \quad f_{31}=1+\sum_{i=2,4}^{\infty} \ldots d_{i} \eta^{i}
$$$$
d_{2}=-1, \quad d_{4}=1 / 4, \quad d_{6}=-1 / 20, \quad d_{8}=1 / 160, \quad d_{10}=-1 / 1440,
$$

etc.;

$$
\begin{aligned}
& I_{31}=-2 C_{1} \int_{0}^{\eta}\left[\left(\eta^{2}-1\right) f_{21} g_{2}-\eta\left(f_{21} g_{2}\right)\right] f_{31} k_{1} \exp \left(-\eta^{2}\right) d \eta \\
& I_{32}=(-1 / 2) C_{1} \int_{0}^{\eta}\left[\left(4 \eta^{2}-1\right) k_{1}-2 \eta k_{1}^{\prime}\right]\left\{2 f_{21} g_{2}-A_{1}^{2} k_{1}^{2} \exp \left[-3 \eta^{2} / 2\right]\right\} f_{31} \\
& \cdot \exp \left(-\eta^{2}\right) d \eta \\
& I_{33}=A_{1} \int_{0}^{\eta}\left[\left(4 \eta^{2}-1\right) k_{1}-2 \eta k_{1}^{\prime}\right] f_{21} f_{31} g_{2} \exp \left(-\eta^{2}\right) d \eta \\
& I_{34}=2 C_{2} \int_{0}^{\eta}\left\{A_{1} k_{1} f_{21} r_{2} \exp \left[-\left(1+B^{2} / 2\right) \eta^{2}\right]+(1 / 2) A_{3}\left(2 f_{21} g_{2}-A_{1}^{2} k_{1}^{2}\right.\right.
\end{aligned}
$$


$\left.\left.\cdot \exp \left[-3 \eta^{2} / 2\right]\right) k_{1} r_{1} \exp \left[-\left(B^{2}+1 / 2\right) \eta^{2}\right]\right\}_{\eta} f_{31} \exp \left[\eta^{2} / 2\right] \eta d \eta$

$$
\begin{aligned}
I_{35}= & -\int_{0}^{\eta}\left\{C_{3}\left(k_{1}^{\prime}-2 \eta k_{1}\right) f_{21} r_{2} \exp \left[-\left(1+B^{2} / 2\right) \eta^{2}\right]\right. \\
& \left.+C_{4}\left[\left(f_{21} g_{2}\right)_{\eta}-\eta f_{21} g_{2}\right] k_{1} r_{1} \exp \left[-\left(B^{2}+1 / 2\right) \eta^{2}\right]\right\}_{\eta} f_{31} \exp \left[\eta^{2} / 2\right] d \eta,
\end{aligned}
$$$$
I_{36}=-C_{5} \int_{0}^{\eta}\left\{\left(k_{1}^{\prime}-2 \eta k_{1}\right) k_{1}^{2} r_{1}^{2} \exp \left[-\left(2 B^{2}+1\right) \eta^{2}\right]\right\}_{\eta} f_{31} \exp \left[\eta^{2} / 2\right] d \eta
$$$$
C_{1}=A_{1}\left(\rho_{0} / \rho_{\infty}\right)\left(\text { inch }^{1 / 4}\right), \quad C_{2}=R \rho_{0} T_{0}\left(u_{0}^{2} \rho_{\infty}\right)^{-1} \text { (dimensionless), }
$$$$
C_{3}=A_{1} G_{21} T_{0} \bar{\mu} \mu_{\infty}^{-1}\left(\operatorname{inch}^{1 / 4}\right), \quad C_{4}=A_{3} G_{21} T_{0} \bar{\mu} \mu_{\infty}^{-1}\left(\text { inch }^{1 / 4}\right)
$$$$
C_{5}=A_{1} A_{3}^{2} G_{31} T_{0}^{2-} \mu_{\infty}^{-1}\left(\text { inch }^{3 / 4}\right) \text {. }
$$

6(b). $p_{31}=C_{8}\left(1+\sum_{i=2,4, \ldots}^{\infty} e_{i} \eta^{i}\right), \quad e_{2}=-9 / 8, \quad e_{4}=63 / 128$

$$
e_{6}=-693 / 5120, \quad e_{3}=6237 / 229376, \quad e_{10}=-39501 / 9175040, \quad \text { etc. }
$$

